\title{
SOBRE UN SUPUESTO ERROR EPIGRÁFICO-HISTÓRICO. CORRECCIONES AL ARA DE BANDUA AETOBRICUS DE CODESEDO (OURENSE) Y A OTROS EPÍGRAFES DE SU AMPLIO ENTORNO
}

\author{
Por \\ ANTONIO RODRÍGUEZ COLMENERO
}

Salida a luz felizmente, esta vez, la segunda edición de Aquae Flaviae I. Fontes Epigráficas ${ }^{1}$, quiero agradecer tanto la cortés felicitación de amigos y colegas como sus observaciones puntuales sobre interpretaciones que pudieran ser discutibles, ya que en epigrafía no abundan las verdades absolutas. Muy recientemente, sin embargo, he tenido conocimiento de una singular y larguísima recensión, en la que, pese a su desproporcionada amplitud, solamente se abordan los epígrafes en que el autor se ve corregido ${ }^{2}$, por supuesto, y lamentablemente, con una imparcialidad de criterio y una elegancia en las formas dignas de peor causa.

Por eso, huyendo de cualquier polémica de tipo personalista y consciente de lo clarificadoras que pueden resultar cara a los lectores algunas precisiones científicas acerca de epígrafes bastante discutidos, alguno de ellos iluminado recientemente con nuevas fuentes de información, todavía no dadas a conocer, intentaré reexplicar pacientemente a mi principal

${ }^{1}$ A. RODRÍGUEZ COLMENERO, Aquae Flaviae I. Fontes Epigráficas da Gallaecia Meridional Interior. Santiago, 1997.

${ }^{2}$ J.C. RIVAS, «Puntualización a unas Fontes Epigráficas». Bol. Aur., XXVII, 1998, $247-272$.

"CUADERNOS DE ESTUDIOS GALLEGOS", Tomo XLVII, Fascículo 113, Santiago 2000. 
interlocutor las razones que me han movido a corregir algunas de sus lecturas, en tanto que, como él bien sabe, he aceptado otras sin reticencia alguna. Pero lo haré, a diferencia de mi oponente, con la representación del documento delante para no confundir al entendido, que de esta manera siempre tendrá ante sus ojos una referencia segura, que pueda permitirle la formación de opiniones, hasta donde ello sea posible, válidas.

\section{Ara de San Vicenzo, Mugares (Aquae Flaviae..., $\mathbf{n}^{\circ}$ 37) (Fig. 1)}

El descubridor de este altar afirma y reafirma que es dúplice, por cuanto posee doble foculus y está dedicado a Júpiter y las Madres, y que las siglas finales han de interpretarse $f($ aciendum) $p$ (osuit).

Sin embargo, el ara sólo tiene de dúplice la división insinuada del campo epigráfico. Lo que determinaría esa duplicidad votiva sería, como en el caso de las estelas, la plasmación de sendos textos independientes en cada uno de los dos sectores. Pero como esto no se hace y, además, no hay manera de leer en dativo matris $^{3}$, clarísimo genitivo complemento determinativo de voto, es por lo que se trata de una vulgar ara unitaria a Júpiter, con paralelos de fórmula de dedicación no demasiado lejanos ${ }^{4}$.

Igual de inviable resulta interpretar $f$ (aciendum) $p$ (osuit), puesto que se trata de fórmulas mutuamente excluyentes cuando se atribuyen a un mismo agente: o se dice $p$ (osuit) of $($ (aciendum) $c$ (uravit). Interpretar dichas siglas como $F$ (lavianus) $P$ (aternus), Fortunatus p(osuit) etc. sería lo más lógico. También, en correlación con el contexto,f(ilius) $p$ (osuit), pero con la contrapartida de permanecer tácito el dedicante. Habrá de transcribirse, por tanto:I(ovi) O(ptimo) Max(imo)/ex voto/matr-is/F(ortunatus)? $P($ osuit $)$ ?, «Fortunato? cumplió el voto que su madre había hecho a Júpiter óptimo Máximo», así de simple.

\footnotetext{
${ }^{3}$ Nos permitimos recordar a J.C. Rivas que el dativo plural de mater es, salvo rarísimas excepciones, matribus, no matris, como parece creer, no sólo por el presente testimonio, sino también por otro derivado de un trabajo suyo anterior (Bol. Aur., 1989) y recomentado también en la presente ocasión, Bol. Aur., XXVII, 1998, pág. 258, al comienzo: «... y luego también ara votiva atribuida a la Civitas Limicorum y a las Matris(sic) Civitatis».

${ }^{4} \mathrm{El}$ más próximo y uno de los más evidentes en la misma provincia de Ourense. Véase $n^{\circ} 77$ bis de Aquae Flaviae..., pág. 107: Cosc(onius) Fl(avinus)/Tutel/(ae) exs/ $\operatorname{vot}($ o) pat(ris).
} 


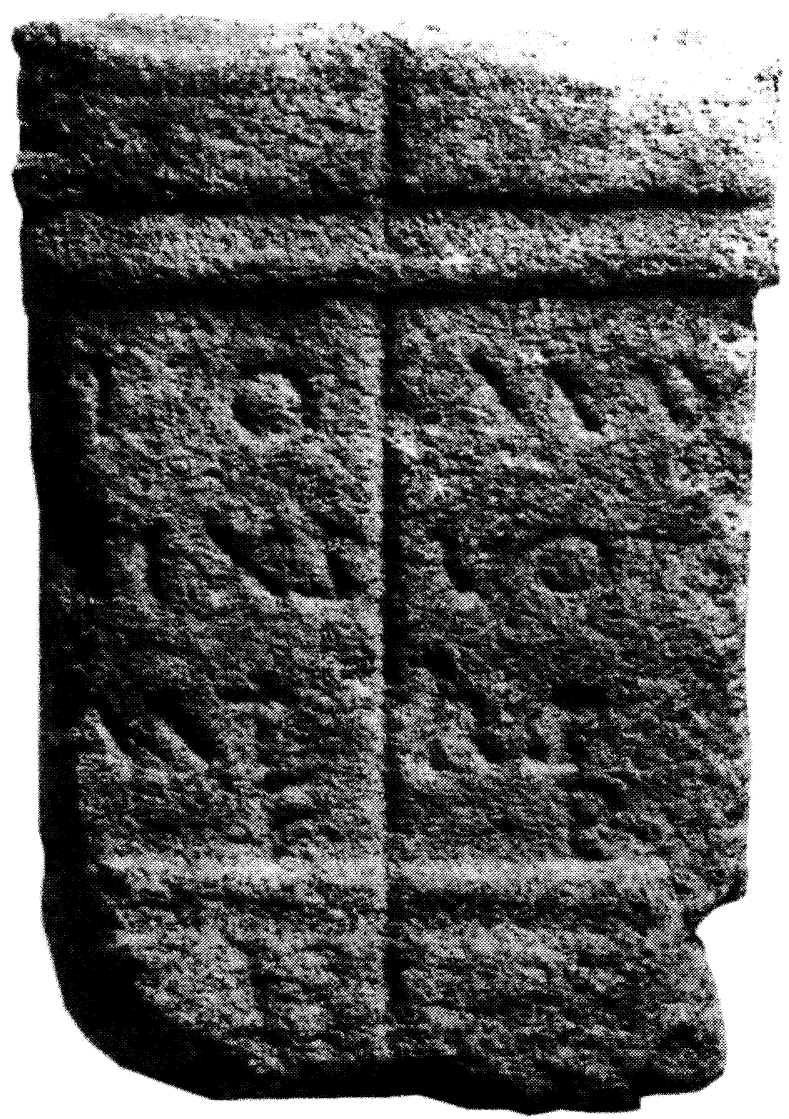

Fig. 1.- Ara de San Vicenzo, Mugares.

\section{Ara de Ponte Bibei (Aquae Flaviae..., $\mathbf{n}^{\circ}$ 10) (Fig. 2)}

El problema reside en las dos primeras líneas. Los autores antiguos leen Iovi La/dico; Tranoy: Iov. O. M. / Digo; Rivas: I(ovi) Oud++/digo, afirmando que existen repicados al final del primer renglón; Caamaño: Iovo La/dico; yo mismo, en la versión última:

Iov-i(nexo $v$-i $) \mathrm{O}($ ptimo $)$ La/dico, señalando que la 1 de Ladico es bífida en la base, que los repicados de Rivas en la primera línea son imaginarios y que la omisión de uno de los dos acostumbrados atributos de la 
divinidad capitolina, en esta caso maximo, no es infrecuente. Y ahora, ante el fotograma y el calco, cada cual puede extraer sus propias conclusiones.

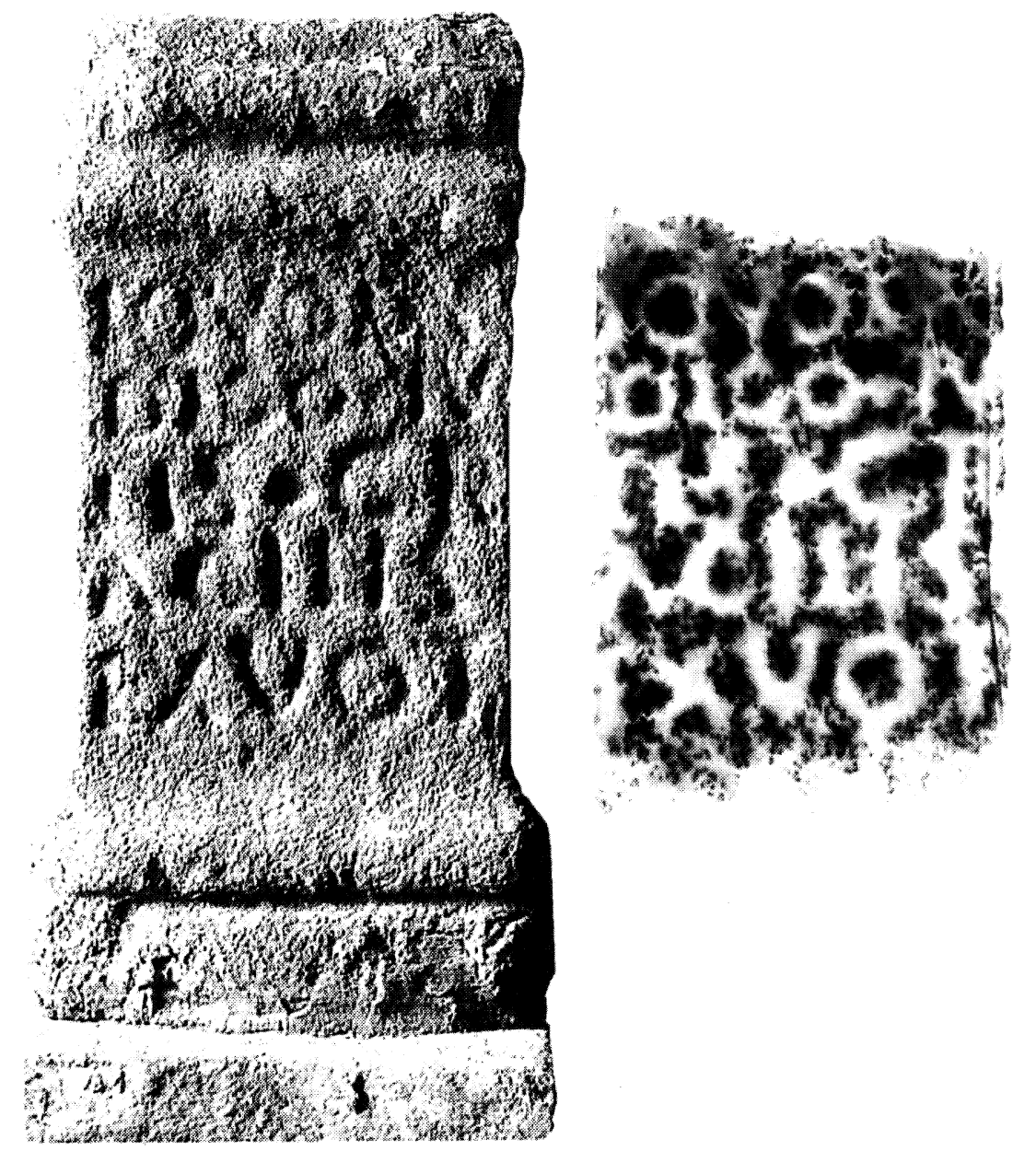

Fig. 2.- Ara de Ponte Bibey y calco. 


\section{Ara de Trasariz (Aquae Flaviae..., $\mathbf{n}^{0}$ 23) (Fig. 3)}

Rivas, el primero en intentar corregir la versión de las $I R G$, $I V$, interpretó en un primer momento, y con un peculiar tratamiento de las concordancias: Iovi Op(timo) Ma/x(imo) ex(actor) de(pulsor) s(olutor) sacrum/ M(arcus) Philippus m(onumentum) a (ram)/posuit.

Poco tiempo después, Le Roux-Tranoy, y tras el encabezamiento, que no ofrece problemas: ...ex de(creto) $s$ (uo)/sacrum/M(arcus) Philippus $\mathrm{Ma}$ (ximus)/posuit, corrigiendo el que suscribe, posteriormente, ex d(creto) por exd(evotione). Rivas está de acuerdo, es la única vez y con reticencias, con las correcciones que se le hacen y me atribuye a mi el mérito, que le devuelvo porque, en justicia, es de los franceses.

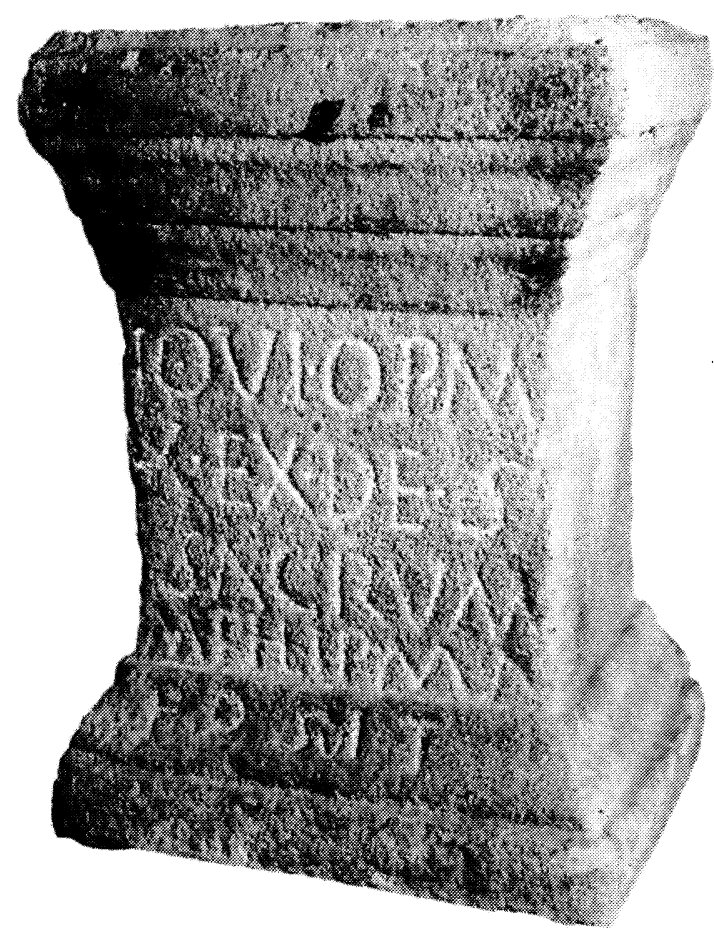

Fig. 3.- Ara de Trasariz.

"CUADERNOS DE ESTUDIOS GALLEGOS", Tomo XLVII, Fascículo 113, Santiago 2000. 


\section{Ara de Eiras, San Amaro (Ourense) (Aquae Flaviae..., $n^{0} 120$ ) (Fig. 4)}

También en este caso la dificultad radica en el segmento del teónimo. Rivas afirma que en el texto se lee Bandu/a Lansb/ricae; Tranoy opina genéricamente, y sin transcribir el texto en detalle, que se trata de una dedicatoria a Bandua Lanobriga y yo mismo, de acuerdo parcialmente con Tranoy, leo, atendiendo a la declinación de los componentes del teónimo, Bandv-e/Alaniob/ricae (nexos $v$-e en el primer elemento y $n-i$ en el segundo). El nexo $v$-e lo había detectado personalmente en un examen del epígrafe efectuado para la primera edición de Aquae Flaviae, examen que,

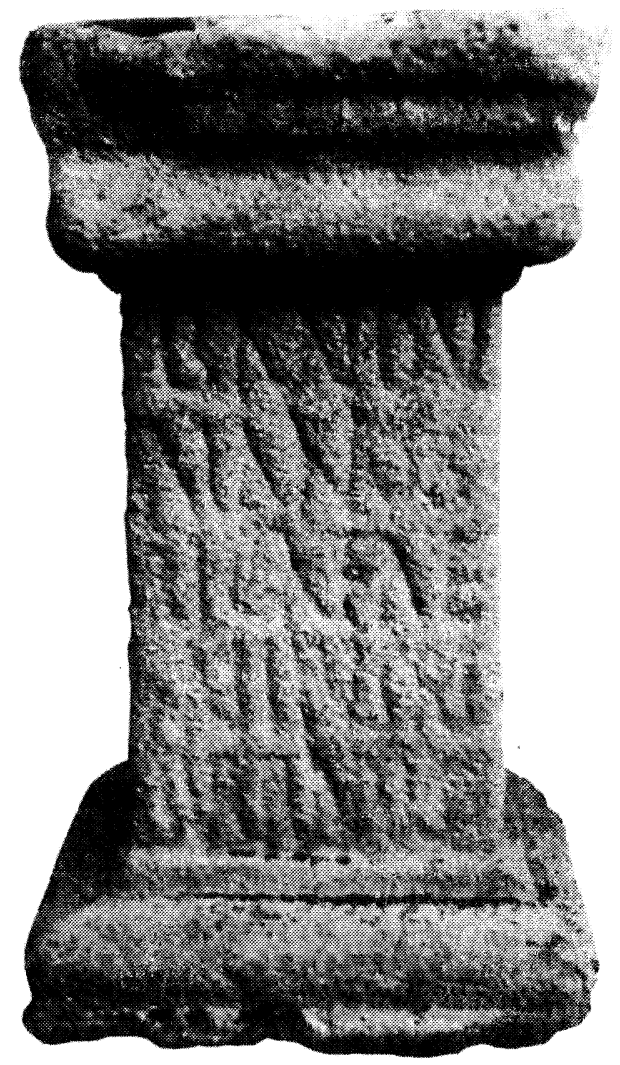

Fig. 4.- Ara de Eiras. 
por razones que desconozco, no me fue permitido por parte del propietario para la segunda. Rivas ironiza con mi creencia de que en este caso se trata de una Bandua femenina (existe, por otra parte, en Extremadura una representación plástica de esta diosa) sin darse cuenta de que Alaniobrigae, para él Lansbricae, no es un topónimo en genitivo, como se deduce de lo que Rivas parece creer, sino un epíteto en dativo concertado con $B a n d u a$, que por esta precisa razón tiene que leerse $B a n d u-(a) e$, no Ban$d u a$, con nexo $v-e$ o con $e$ apocopado en la forma $B a n d u(e)$, si bien personalmente estoy convencido de lo primero ya que en la foto misma se vislumbra dicho nexo. Esta es, por otra parte, la función y concordancia que se advierte en los paralelos conocidos; por citar sólo los más próximos, en Bandue Verubrico, Bandue Cadaego, Bandu-e Vei-gebreaego (nótese el nexo v-e) y Bandu-e Vordeaeco (también con el mismo nexo). En todo caso, la gran S, clarísima para Rivas, de Lansbricae, se convierte en una O suficientemente nítida para Tranoy y para mi.

\section{Placa funeraria de Codesedo, Sarreaus (Ourense) (Aquae Fla- viae..., $\mathbf{n}^{0}$ 224) (Figs. 6 y 7 )}

\subsection{Limitaciones de partida}

No he podido examinar la pieza por no haberla encontrado en donde Rivas la situaba. Al parecer, había sido adquirida por su mediación y no se hallaba todavía expuesta en el Museo Provincial en el momento de redactar este estudio. La fotografía del epígrafe, con letras indebidamente repintadas, no cabe dudar que por su descubridor, así como el diseño facsímil de los que nos hemos valido, son reproducción de los publicados por Rivas en su trabajo. Ello, sin embargo, no alterará, en lo esencial, nuestras conclusiones.

\subsection{Interpretación del epígrafe}

Rivas: Ale[civs (?). B]/ibali. f(ilius). /V(icus). /Nemetobrica. I H(ispaniae) N(ovae) C(iterioris) (Antoninianae). Sit [te(erra) l(evis).

Colmenero, y sólo en la hipótesis de interpretar $V$. por Vicus, lo cual parece probable, aunque no probado, tres posibilidades: 


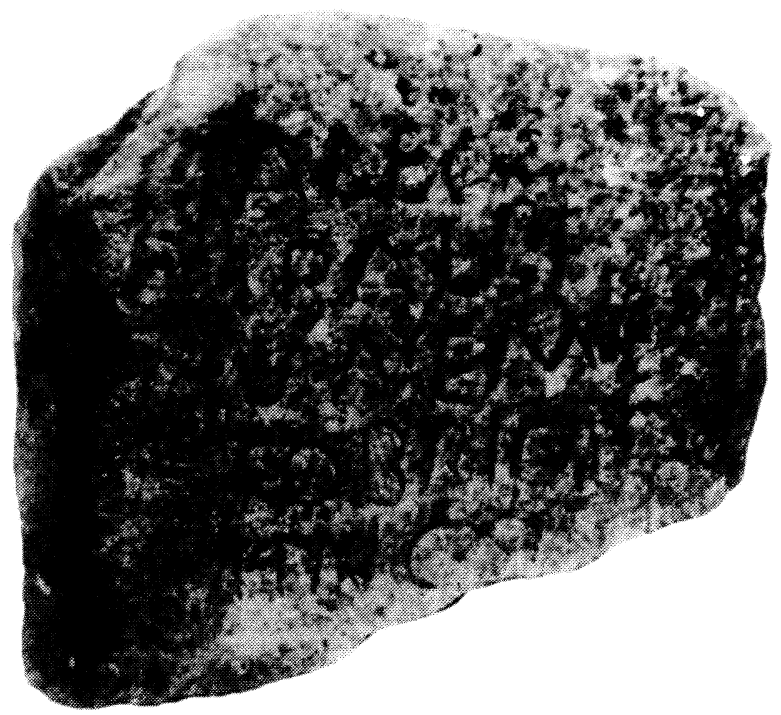

Fig. 6.- Placa funeraria de Codesedo, Sarreaus (Ourense).

- En el caso de que sólo fuese posible leer Nemetobrica, como quiere Rivas, y no Nemetobricae:

Alec[io B]ibali f(ilio)./V(icus).Neme/tobriga./h(ic)?n(on)? c(ubat)?. sit t(ibi) t(erra) [l(evis)]. «El poblado de Nemetóbriga hace esta dedicatoria a Alecio, hijo de Bíbalo, pese a que no está enterrado aquí. Que la tierra te sea leve». La objeción posible es que, mientras se conoce la fórmula $h(i c) c(u b a t)$, no está constatada, al menos que yo sepa, la fórmula $h($ ic) $n($ on) $c$ (ubat), que vendría a expresar lo contrario, y también lo contrario de $h($ ic) $s$ (itus) $s($ est $)$; por eso cabría la posibilidad de un epitafio «in absentia», práctica sobradamente conocida en la epigrafía del Imperio.

- Si pudiese leerse Nemetogrica-e (nexo a-e), como sospecho, habrían de ser los v(icani) Nemetobrigae los dedicantes, lo que resulta más usual. Lo demás, idéntico. En ambos casos, y al tratarse de una dedicatoria colectiva, el vicus Nemetobriga habría de localizarse, casi necesariamente, en las inmediaciones del lugar de la dedicatoria y, por tanto, dentro del ámbito de la civitas de los Limici. 


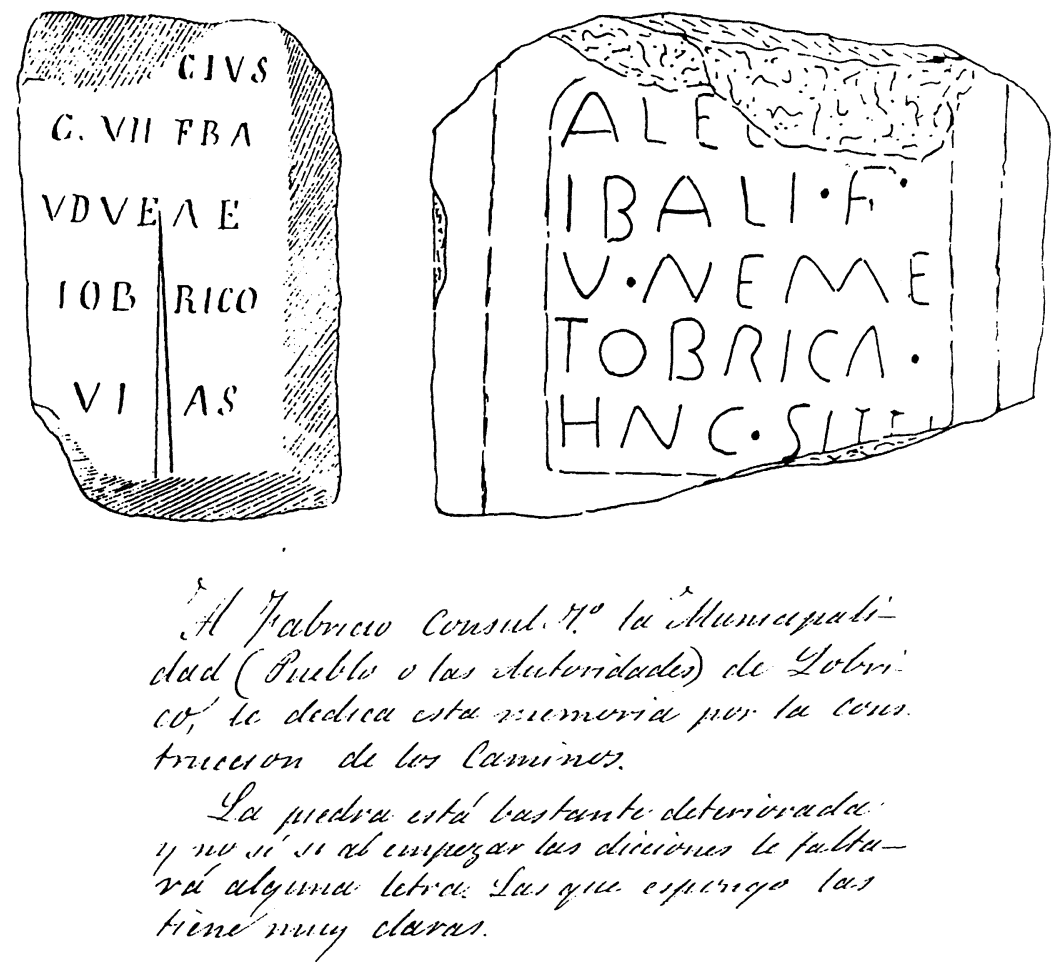

Fig. 7.- Ara de Barros y estela de Rivas. Contraste entre ambos diseños.

- Alec[io B] ibali f(ilio)/v(icano) Nemetobrigae(vale también el ablativo) $H ? N ? C$ ? (siglas del o los dedicantes) Sit t(ibi) t(erra) [l(evis)]., «H?N?C? (dedicantes) hace, o hacen, esta dedicatoria aAlecio, hijo de Bíbalo, oriundo de Nemetobriga. Que la tierra te sea leve». En este caso, sí que la Nemetobriga de la inscripción podría corresponder a la mansión viaria tibura.

Que existan núcleos urbanos con el mismo nombre, aún poseyendo distinta categoría, es lo más normal, sin necesidad de «clonarlos», como, con poca fortuna, quiere ironizar Rivas. Por ejemplo, entre los Astures existen dos poblaciones llamadas Interamnium y dos Talábrigas, de condición jurídica diferente, en la franja occidental: la civitas lusitana y la unidad precedida de $c$ invertida de los Límicos, precisamente.

"CUADERNOS DE ESTUdios GALLEGOS", Tomo XLVII, Fascículo 113, Santiago 2000. 
En contrapartida, ¿por que considero poco viable la transcripción de Rivas? Razones obvias me llevan a ello ya que, aún leyendo v(ico) Nemetobriga, en ablativo, como lugar de procedencia, y no en nominativo, como él hace, volviendo así imposible la interpretación del conjunto, sobraría ese innecesario vico a la hora de designar la, en este caso, mal llamada origo y habría que atribuir las funciones de sujeto a la hipotética Hispania Nova Citerior (Antoniniana), que ni el texto (se escribiría, en tal caso $P$ (rovincia) H(ispania) N(ova) C(iterior) A(toniniana) o H(ispania) N(ova) $C$ (iterior) $A$ (ntoniniana $^{5}$, ni la práctica epigráfica a la hora de señalar la procedencia de un emigrado (aún forzando las cosas se recurriría, por encima del vicus, a la civitas, pero jamás a la provincia), ni el mismo contexto histórico, sumamente problemático, en que se inserta esa, más desconcertante que efímera, provincia sobre la que existen novedades de última hora que en breve va a dar a conocer G. Alföldy ${ }^{6}$, aconsejan.

5.3. ¿Es posible identificar el presente epígrafe con el ara a Bandua descubierta por Barros Sivelo en las cercanías de la iglesia de Codesedo (CIL, II, 2515), según él mismo Barros afirma (Antigüedades de Galicia, La Coruña, 1975, 181)?

Tras el hallazgo de la inscripción que acabamos de comentar, Rivas ha querido ver en ella la que Barros dio a conocer mediada la segunda mitad de la pasada centuria ya que, razón esencial, la procedencia es practicamente la misma. Esto le llevó a publicar un artículo bastante extenso, enfáticamente titulado «Un error epigráfico histórico: el supuesto dios galaico-romano Bandueaetobrigus» (CEG, XXIX, 104, 1991, 35 ss.), negando que tal divinidad, tan traída y llevada por los estudiosos de las religiones antiguas, hubiese existido en otro lugar que no fuese la defectuosa transcripción efectuada por Barros y casi simultáneamente maquillada por Hübner para incluirla en su CIL, II. Pese a que contrasta ambos textos, en realidad en muy pocas cosas parecidos, halla fácil explicación para las discrepancias y, como no, concluye sancionando tal identificación.

\footnotetext{
${ }^{5}$ CIL, II, 2661, 5680; CIL, VI, 41229.

${ }^{6}$ Mientras tanto puede consultarse G. ALFÖLDY, «Der Status der Provinz Baetica um die Mitte des 3. Jahrhunderts», Festschrift für Hans Lieb. Roma, 1995, 29-42.
}

"CUADERNOS DE ESTUDIOS GALLEGOS", Tomo XLVII, Fascículo 113, Santiago 2000. 


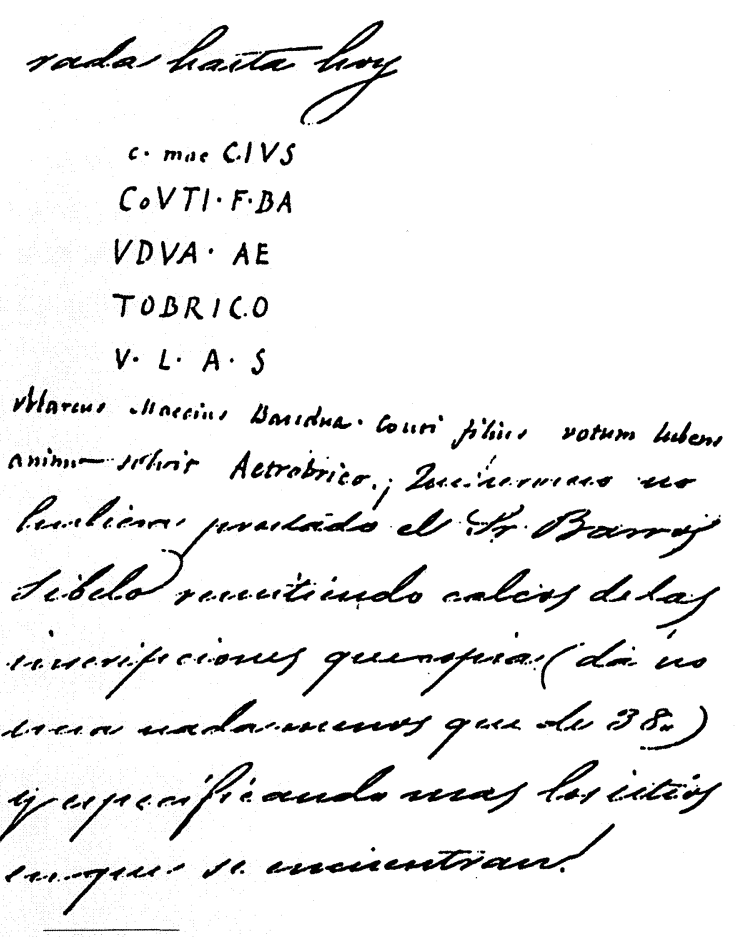

Fig. 8.- Ara a Bandua Aetóbrigo. Primera reinterpretación de Hübner sobre diseño de Barros.

(Misiva de la Academia de la Historia a Barros).

En lo que a mi respecta, en absoluto me convencieron nunca las razones de Rivas y manifesté mi punto de vista en contra en un trabajo posterior (Aquae Flaviae..., 1997, 144). Me parecían irreductibles las diferencias existentes entre ambas leyendas, aún concediendo que Barros pudiese haber leído mal. Esta opinión no pareció sentar bien a nuestro oponente y trató de fustigarla, con más pasión que razón, en el intento de recensión antes mentado. Por nuestra parte, contamos para la presente ocasión con datos definitivos que pueden contribuir a zanjar la cuestión, entre ellos el diseño original del cipo votivo ejecutado por Barros, que mostramos al lado del boceto que Rivas hace de la placa por él descubierta (Figuras 6 y 7), y pasajes poco conocidos de la memoria que Barros adjunta a su plano de la vía XVIII (Figuras 8 a 11). Del contraste entre ambas inscripciones, podemos deducir las siguientes sustanciales diferencias:

"CUADERNOS DE ESTUDIOS GALLEGOS", Tomo XLVII, Fascículo 113, Santiago 2000. 


\title{
CIL, II, 2515 En Ginzo de Ltmia.
}

\author{
... CIVS \\ $\mathrm{C} \cdot \mathrm{VII} \cdot \mathbf{P} \cdot \mathbf{B A}$ \\ VDVEA E \\ Barros Sib \\ IOBRICO \\ traditar. \\ brigus, cf. \\ s $\mathrm{V} \cdot \mathrm{LA} \cdot \mathrm{A}$
}

Fig. 9.- Misma transcripción de la Fig. 7, y segunda interpretación de Hübner.

- Las siluetas de ambos objetos difieren totalmente, contando el diseño de Barros con una contramarca angular en el centro inferior, que en modo alguno se descubre en el otro, enmarcado entre gruesos verdugones verticales; $y$ ello sin tener en cuenta que las zonas erosionadas del inicio se hallan en lugares contrapuestos.

- Los textos se asemejan exclusivamente, y sólo en parte, en la cuarta línea. Por el contrario, la quinta los define, a uno como inscripción votiva y al otro como funeraria.

- No es verdad que Hübner alterase tanto la transcripción de Barros; lo que hizo, al menos en su versión definitiva ${ }^{7}$, según puede com-

\footnotetext{
${ }^{7}$ Porque lo que no puede ignorar nuestro interlocutor es que Hübner, a requerimiento de los miembros de la Academia, de la que formaba parte como Correspondiente, dio primero la versión c. mae CIVS/CoVTI. F. BA/VDVA. AE/TOBRICO/V. L. A. P., interpretando Marcus Maecius Bandua, Couti filius, votum libens animo solvit Aetobrico (GAYANGOS et allii, «Sobre los trabajos de Fray Pedro Cid y del Señor don Ramón Barros Sibelo para ilustrar el segundo camino de Braga a Astorga», BRAH, I, 1877, 179 ss. (Fig. 8), aunque en el manuscrito del informe de Gayangos y Compañía no constaba el nombre de tan ilustre intérprete y constan, por el contrario, muchos más comentarios sobre Barros que los que refleja el informe publicado) si bien posteriormente (CIL, II 2515) recogió velas y se ciñó estrictamente a lo que Barros transcribió, interpretando esta vez Bandvae Aetobrico como teónimo compuesto (Fig. 9).
}

"CUADERNOS DE ESTUDIOS GALLEGOS", Tomo XLVII, Fascículo 113, Santiago 2000. 


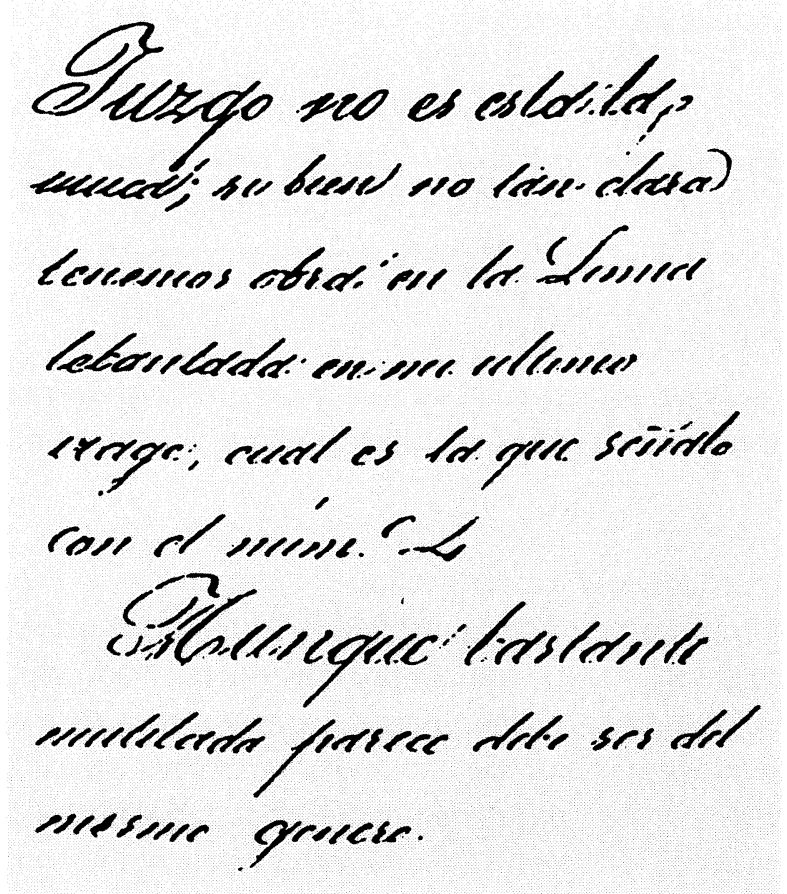

Fig. 10.- Lugar de hallazgo de la inscripción $n^{\circ} 4$ de Barros.

probarse, es volverla interpretable (Fig. 8), ciñéndose estrictamente a los rasgos transmitidos por Barros, cuyo diseño también él conocía. Barros, por su parte, prendido de la lectura del último renglón, para él VIAS, en vez de V(otum) L(ibens) A(nimo) S(olvit), había aventurado una traducción ciertamente disparatada, aunque no exenta de lógica en algunos segmentos ${ }^{8}$. Sin embargo, la fidelidad de su transcripción la remarca en el segundo párrafo a pie de diseño, que también ofrecemos al lector Fig. 7).

${ }^{8}$ Barros traduce la C. de la segunda línea por cónsul y VII por un numeral, señal de que lo vio así, puesto que corrobora la fidelidad del texto al afirmar: «... las (letras) que expongo las tiene muy claras. Eso intuí yo también en ocasiones anteriores al leer [le]gIonis) VII F(elicis), válido para ese segmento de haber estado más confuso el inicio». Tras el rescate del diseño original ya no sé que pensar.

"CUADERNOS DE ESTUdios GALLEGOS", Tomo XLVII, Fascículo 113, Santiago 2000. 
- Rivas dice haber extraído su inscripción de las paredes de una construcción sin techo; Barros, por el contrario, afirma (pág. 4 de la respuesta a los requerimientos de la Academia) que «... si bien no tan clara, tenemos otra en la Limia levantada en mi último viaje, cual es la que señalo con el número 4» (o sea, la que mostramos en el texto facsímil). Por tanto, levantó de la tierra su lápida, poniéndola de pie, como, frecuentemente, dice que hacía con los miliarios de la vía.

\subsection{Item más, la inscripción de Barros no fue hallada en Codese- do, aunque se nos ocultan las razones que le movieron a afirmar esa procedencia}

Ya nos extrañaba que Hübner, que tuvo acceso directo a su plano y comentarios, prescindiese de lo afirmado por Barros en sus Antigüedades, y atribuyera el epígrafe a «Ginzo de Limia» y no a Codesedo; y que bastantes años después, Fita mismo tampoco se refiriese a Codesedo como lugar del hallazgo y diga, en cambio, que fue encontrada en el «Campo de Limia» ${ }^{9}$. Y es que lo de Codesedo no lo afirmó Barros nunca antes de su tardía publicación de las Antigüedades de Galicia. Acabamos de ver en el párrafo transcrito en la Figura 10, y que ofrecemos en facsímil, que dice haberla encontrado en La Limia, pese a contestar al apremiante requerimiento de la Academia para que concrete el lugar de sus hallazgos, auténtico talón deAquiles de su proceder, que le estarán reprochando continuamente. $\mathrm{Y}$ añade en la página 11 , al contestar a la quinta pregunta de la encuesta a la que se ve sometido, «que desde la aldea de Zadagós la vía mencionada según mis investigaciones se dirige a Baños de Molgas (Gemina). No ha duda de que el puente de Arnuiz es obra Romana, pero no perteneciente a la vía de que se trata, que es la que comunicaba los pueblos de la margen izquierda del Lago (los Lémicos) con ella confluyendo en el Monte del Medo. Comprueba esta idea no sólo la situación topográfica sino una lápida encontrada en esta zona de camino y que descubrí el año pasado y que señalo con el número 4» (vide facsímil Fig. 11). Más

\footnotetext{
${ }^{9}$ F. FITA, «Lápidas romanas de Mosteiro de Ribeira, feligresía de Ginzo de Limia en la provincia de Orense», BRAH, LXVIII, 388 ss. (1911). Sin embargo, la interpretación que Fita realiza de la inscripción supera negativamente a la que Barros ofrece, no comprendiéndose cómo este insigne epigrafista la considera dedicada a Ceres.
}

"CUADERNOS DE ESTUDIOS GALLEGOS", Tomo XLVII, Fascículo 113, Santiago 2000. 


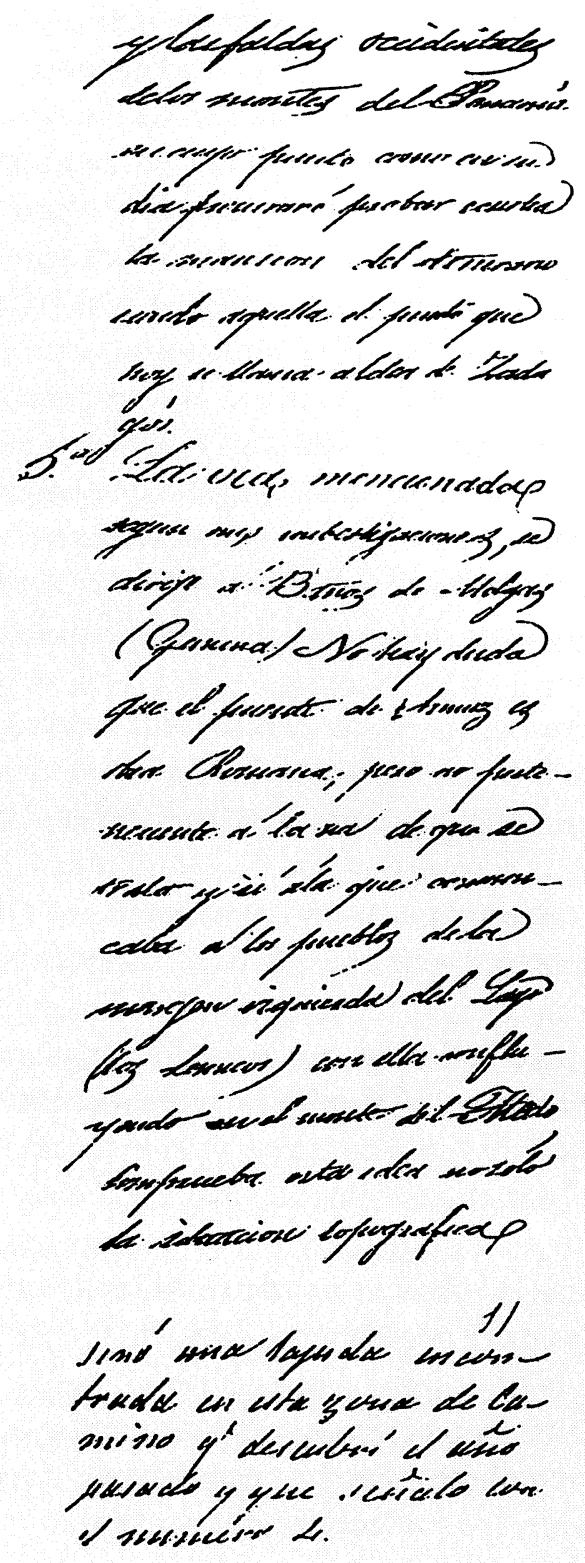

Fig. 11.- Texto de Barros, extraído de su informe a la Academia de la Historia, relativo al lugar del hallazgo de su inscripción $n^{\circ} 4$. 
claro, imposible. Pero ¿por qué oculta Barros la verdad? No lo sabemos, mas pudieran barajarse dos hipótesis. Primera, que al escribir sus Antigüedades de Galicia sufriese un lapsus y confundiese Codesedo, a más de quince kilómetros al sur del decurso de la vía, en donde a Barros no se le perdía nada con respecto a su estudio, con Sobradelo, ubicado precisamente en ese tramo descrito en último lugar. Segunda que, consciente del aluvión de críticas negativas que en la Academia habían surgido ante la impresentable traducción que había realizado del epígrafe, pretendiese ocultar su verdadera ubicación para no sufrir nuevos ataques del Sr. Fernández Guerra (la tensión entre ambos se percibe en la lectura de todo el proceso), a la sazón responsable de trabajos geográficos muy próximos a la zona de estudio de Barros y que, junto con Saavedra, aprovechó los datos suministrados por el monumental mapa del autor corunés para fijar bien y definitivamente las mansiones de la vía desde Bracara hasta Salientibus, ubicando, por primera vez correctamente, la mansión de Aquis Querquernis.

Tras lo dicho, no existe razón para borrar del mapa teonímico a Bandua Aetobrigus, como Rivas ha hecho, o, por lo menos, antes de que sean alegadas razones convincentes; ni siquiera existen argumentos definitivos para no poder transcribir en la segunda línea [le]g(ionis) VII $F$ (elicis), tanto más cuanto que Barros transcribió el VII, en su peculiar versión, como un numeral, según puede observarse en la Fig. 7. De todas maneras, y aún no siendo un soldado el dedicante, la relación de Bandua con la actividad bélica vendría sugerida, en primer lugar, por la dedicatoria de Marcus Silonius Silanus, un signifer, a Bandua en Rairiz de Veiga y, en segundo término, por la perdida inscripción de Deo Vexillorum/Martis Socio/Banduae, sintácticamente correcta, transmitida por todos los autores antiguos y que no se puede dar por falsa, a menos que se den por falsas todas las que fueron transmitidas del mismo modo. En todo caso, la concordancia temática, signifer, de la inscripción conoci$\mathrm{da}$, y vexillorum, de la hoy perdida, atribuidas a un mismo lugar de procedencia, hablan de la protección que esta divinidad dispensaba a los colectivos militares cobijados bajo los respectivos signa y vexilla, divinidad que, lógicamente, los soldados romanos asimilarían a su dios de la guerra.

"CUADERNOS DE ESTUdios GALLEGOS", Tomo XLVII, Fascículo 113, Santiago 2000. 


\section{Estela votiva de Nocelo da Pena, Xinzo de Limia (Ourense) (Aquae Flaviae..., $\mathbf{n}^{0}$ 172) (Figs. 12 y 13)}

Que la pieza tiene forma de estela bifronte, al parecer el gran descubrimiento de Rivas, nadie lo duda ni lo han dudado los que le precedieron aunque, a veces, no lo digan con las mismas palabras, como, por el contrario, nadie duda de que el epitafio de Iulius Rufinus Leontius, del Museo de Lugo, se ha plasmado sobre un ara, en principio monumento votivo. Pero ¿qué es lo que define el carácter votivo o funerario del monumento?, el texto mismo. Y en descifrar ese texto, ciertamente difícil, pero recuperable, nos hemos afanado bastantes investigadores, intentando hipótesis verosímiles, después superadas por otras más ajustadas. Así avanza la investigación epigráfica, que ha de estar siempre desligada de argumentos de autoridad y dogmas preestablecidos.

Tratando de ahorrar disquisiciones inútiles, ya avanzadas en trabajos anteriores, y a la vista de lo que se percibe en los fotogramas de anverso y reverso que ofrecemos, propongo personalmente la siguiente lectura:

- Anverso: $M$-a-tri(bus) civita(tis)[pr(o)/A]m-ac(a)e Avi[t(a)e s(alute)]Taciu/s et Ta[pila].
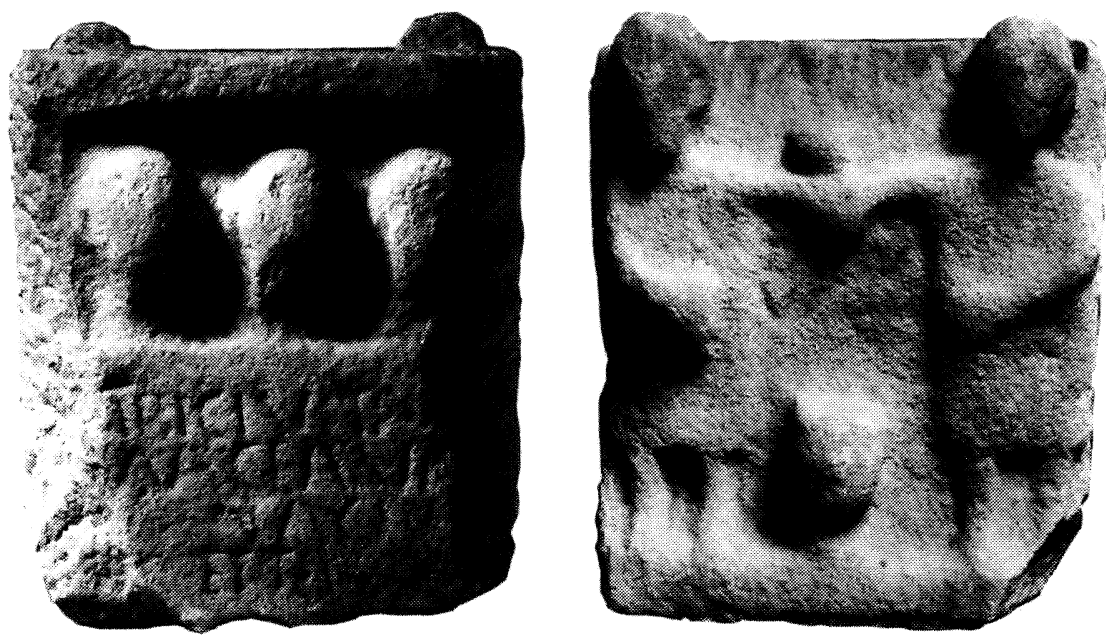

Fig. 12.- Estela votiva de Nocelo da Pena, Xinzo de Limia (Ourense).

"CUADERNOS DE ESTUDIOS GALLEGOS", Tomo XLVII, Fascículo 113, Santiago 2000. 
Tacio y Tapila ofrecen este altar a las Madres de la civitas (de los Límicos) por la salud de Ámaca Avita.

\section{- Reverso: Tapila/(et) Taciu[s]/v(otum) s(olverunt) l(ibentes) [m(erito)].}

Tapila y Tacio cumplieron su voto con ánimo complacido.

Rivas, sin embargo, se siente molesto e incomprendido porque algunos rehusamos adherirnos a su parecer, y tacha a Tranoy, con una versión también diferente, de orgulloso o algo así y a mi poco menos que de iluso (CEG, XXXIX, 104, pág. 44, nota 23). Como alternativa, y aunque protesta de que los rasgos son borrosos y poco descifrables, y siempre partiendo del axioma de que se trata de una inscripción funeraria, propone, no como ahora afirma «exclusivamente a modo ideográfico, sin pretender leer el texto realmente grabado» (Puntualizaciones, 258), sino como «una de las redacciones posibles» (Sobre la identidad..., pág. 92), «o sea que

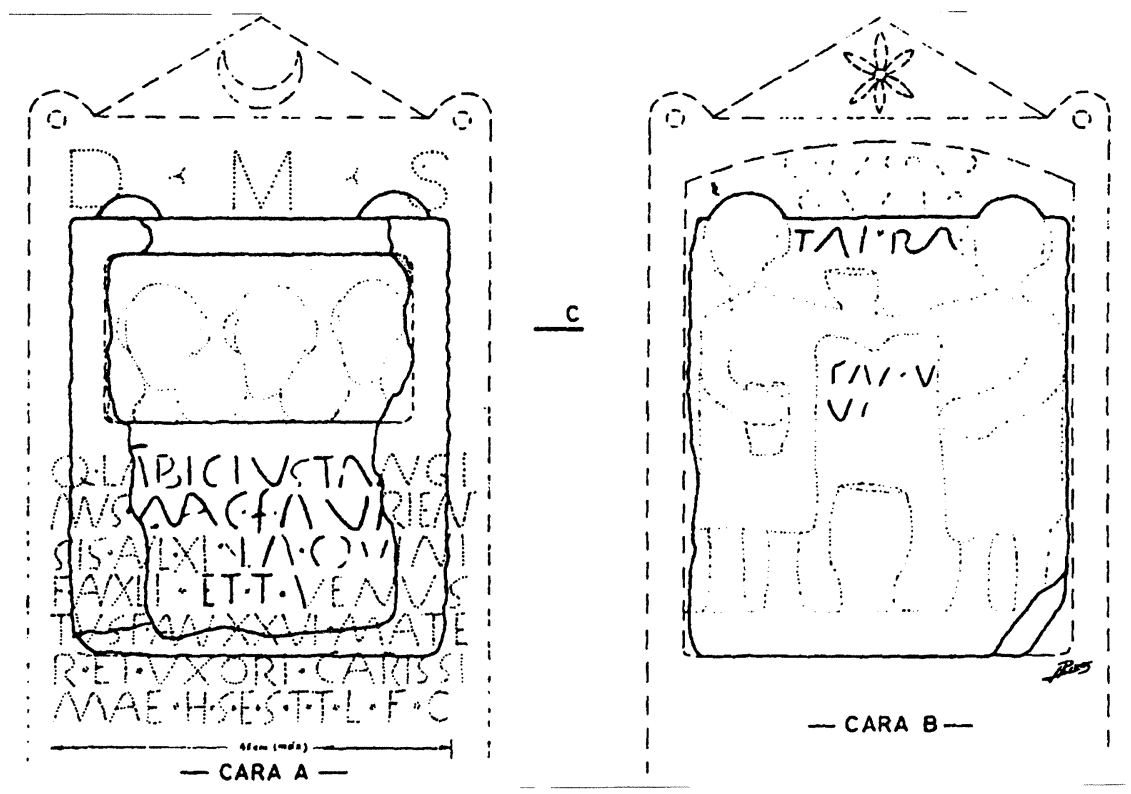

Fig. 13.- Reconstrucción ideal de Rivas.

"CUADERNOS DE ESTUDIOS GALLEGOS", Tomo XLVII, Fascículo 113, Santiago 2000. 
nos acercamos más al texto real con una redacción del tipo que indicamos en nuestro dibujo, así (Idem..., pág. 94): [Q(uintus) L] abicius Ta[ngi/nus] M-ac(rini). f(ilius). Aur[ien/sis. a-n(norum) LXI.] La(bicio). Ov[ni(us)/ f(ilio) a-n (norum) XLI] et T(itus). V[enustus/f(ilio) an(norum) XXVI. mater et uxori carissi/(mae). h(ic). s(itus). e(st). s(it) t(ibi). t(erra). l(evis). $f$ (aciendum) c(uravit)».

Como puede observarse, en esta versión no falta casi nada, más bien sobra. Lo único que sugeriría al Sr. Rivas es que, ya que algunos nos declaramos impotentes para acometer tal empresa, busque quien se la traduzca coherentemente tal como está (constituye un excelente rompecabezas para iniciados), haciéndonoslo saber cuando lo consiga ${ }^{10}$.

\section{Ara a Júpiter de la basílica visigótica de Santa Comba de Bande (Aquae Flaviae..., $\mathbf{n}^{0}$ 22)}

Rivas repite ad nauseam (Puntualizaciones..., 251, nota 1) que se trata de la misma que mencionan una retahíla de autores antiguos de todos conocidos y que dan como dedicada a los Lares Viales, con un texto para nosotros correcto y convincente (vide Fig. 14). Sin embargo, y contradictoriamente, añade que la actual de Sta. Comba no está dedicada a los

\footnotetext{
${ }^{10}$ Digo «coherentemente» porque, tal como Rivas estructura su texto latino, habría que traducir: «Quinto Labicio Tangino, hijo de Macrino, natural de Ourense, de 61 años de edad a Labicio; Ovinius a su hijo de 41 años y Tito Venusto a su hijo, de 26 años. La madre y para la esposa carísima. Está enterrado aquí. Que la tierra te sea leve. Procuró hacerlo». De esta manera, los años se le adjudicarían al vivo y no al muerto en el primer caso, no se conocerían los nombres del segundo y tercer difuntos, pero sí, curiosamente, sus edades, no se sabe que pintan en el lugar una madre (¿de quién?) y una esposa y, al final, con tantos individuos muertos, solamente aparecería uno enterrado, eso sí, bien enterrado a juzgar por la redundancia de las fórmulas.

Por otra parte, y aún desde el punto de vista escultórico, la reconstrucción «ideográfica y conjetural» que Rivas hace de la parte superior de la estela (Fig. 13) resulta inverosímil, ya que uno no se explica, si se fracturó accidentalmente, porque ofrece un perfil tan regular y rectilíneo, con dos cabezas asomando, simétricamente dispuestas, y si fue recortada intencionadamente para readaptarla a un lienzo de muro, por ejemplo, por qué se respetaron esas mismas cabezas descollantes. Tales objeciones hacen suponer que la parte superior de la estela votiva siempre fue así, por lo que las idealizaciones de Rivas resultan poco creíbles.
}

"CUADERNOS DE ESTUDIOS GALLEGOS", Tomo XLVII, Fascículo 113, Santiago 2000. 
Lares Viales, pese a que es la misma ${ }^{11}$. Nos gustaría que se aclarase antes de imputarnos la publicación doble de un mismo texto, que, en absoluto se ha hecho en esta ocasión. En todo caso, lo esencial es la lectura de una inscripción, hoy existente en el interior de la basílica de Santa Comba, que presentamos con foto y calco (Fig. 15) y que Rivas da como ininteligible. Partiendo del hecho cierto de que la tercera letra de la primera línea, una $V$, posee su primera barra cruzada por una hendidura transversal accidental, lo que inicialmente genera una cierta confusión, ningún epigrafista que se precie de tal podrá dejar de leer, al menos, Iovi en el primer renglón, Optum(o) en el segundo, $M$-ax(imo) en el tercero, Asiat(icus) en el quinto y $P$ (osuit) $V$ (otum) $L$ (ibens) en el sexto. Si Rivas no llega a tanto es su problema.

Y llegados a este punto, no hay mucho más que añadir porque el especialista ya se habrá formado una idea aproximada de la credibilidad que cada cual merece y seguir discutiendo sobre el texto de unos miliarios

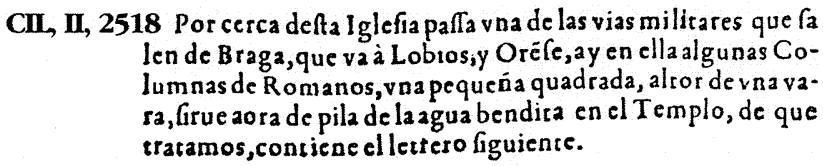

$$
\begin{aligned}
& \text { MAX SV } \\
& \text { MVS-IOV } \\
& \text { ESSI-F-LA } \\
& \text { RIBVS V I } \\
& \text { A LIB V S } \\
& \text { V.S. L. M. }
\end{aligned}
$$

Fig. 14.- Ara a los Lares Viales (Castellá Ferrer), hoy día extraviada.

\footnotetext{
${ }^{11}$ Una razón complementaria por la que no puede ser la misma es que Castellá Ferrer el primero en darla a conocer (Fig. 14), afirma que servía de pila de agua bendita y la que ahora ofrecemos (Fig. 15) no presenta vaciado para tal fin, según puede comprobarse «in situ». Vide texto que acompañaba al ara y que ha sido tomado de la obra de Castellá en la Figura 14.
} 


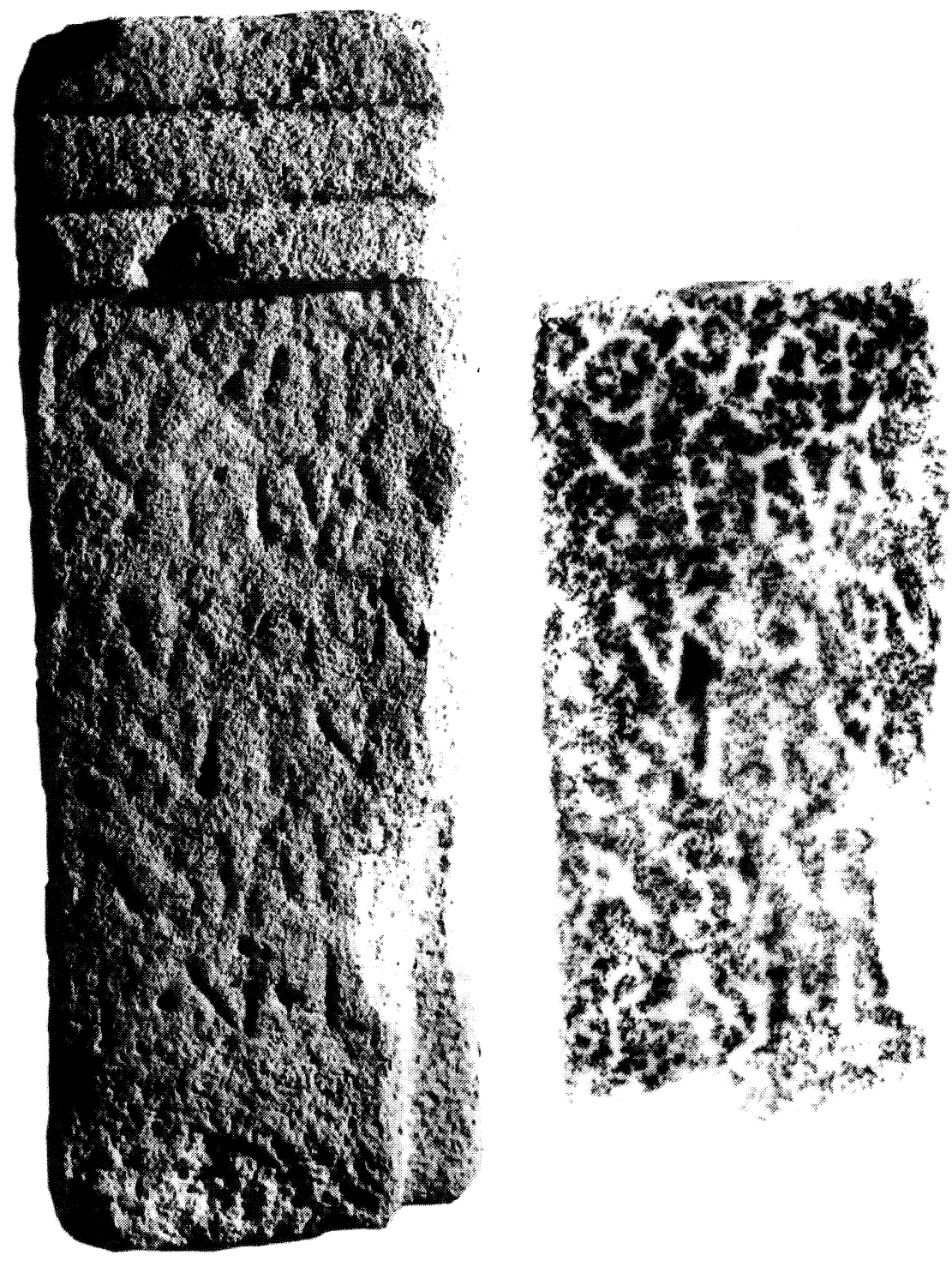

Fig. 15.- Ara a Júpiter de la basílica visigótica de Santa Comba de Bande, con el calco respectivo. 
que, por la peculiar ordinatio circular de su texto, no resultan comprobables a no ser mediante un derroche de fotografías, resulta totalmente estéril. Sólo puntualizaré a nuestro interlocutor, antes de concluir, que, con respecto al ara de Moreiras (Aquae Flaviae, $n^{\circ} 141$ ), esas cuatro vocales seguidas del epíteto Proeneiaeco «imposibles e inadmisibles» en latín, para Rivas, se ven superadas en número, pongo por caso, por las cinco seguidas del verbo eieiulare ¿lo conoce nuestro oponente?; que seguir empeñándose en leer al final del primer renglón de la dedicatoria de Asadur vec(tigalium) II(viro) equivale a saber muy poco de la naturaleza de los vectigalia, de la función de los duoviri y de las pares rationes conseguidas del fiscus imperial, que en la inscripción se mencionan; y, finalmente, que los muy rudimentarios medios para la medición empleados por el que suscribe en las lejanas datas de 1972 para el tramo viario de Portela d'Home sirvieron para establecer, por primera vez, una longitud de milla de $1.666 \mathrm{~m}$. (+-) que, al menos para aquel tramo, ha sido refrendada posteriormente por Caamaño y recientemente por Santiago Ferrer.

\section{POST SCRIPTUM}

Cuando estaba a punto de salir de imprenta el presente número de Cuadernos nos fue comunicado, por el Arqueólogo Provincial de la Delegación de Cultura de Ourense, don Alfredo Seara,que había sido descubierta en Codesedo, al derrumbarse un muro, el ara dedicada a Bandua, de la que hemos hablado extensamente al abordar la inscripción número cinco, siendo don J. Carlos Rivas mismo quien puso sobreaviso a las autoridades competentes. Se comprenderá que la oportunidad del hallazgo me haya obligado a añadir esta nota, agradeciendo al director del Museo de Ourense las facilidades ofrecidas para fotografiar el epígrafe.

Se trata de la misma inscripción a Bandua que Barros Sibelo había dado a conocer, y casi como él la había dado a conocer. Las diferencias consisten en la sustitución de $\mathrm{C}$ seguida de punto, por una $\mathrm{A}$, en el inicio del segundo renglón, y la de VIAS (caminos, para Barros), en la línea final, por la conocida fórmula $V$ (otum) $S$ (olvit) L(ibens) M(erito), que ya Hübner había recompuesto en su momento. El sector inferior,sin embargo, se muestra menos completo que cuando Barros lo describió. Por otra parte, el epíteto de la divinidad es algo parecido a Aediobrigo (sea $d$ u otra 
letra la que se halla erosionada en el tercer renglón), parecido a lo que Barros había transcrito, y no Aetobrigo, como interpretó Hübner y los que posteriormente le hemos seguido. En resumen, a la vista del original podría transcribirse: [-_cius? [FI]/avii f(ilius) Ba/ndue Ae[d?]/iobrico/v(otum) l(ibens) a(nimo) s(olvit).

Es para congratularse de que se haya rescatado para la ciencia epigráfica la conocida dedicatoria a Bandua y de que, al menos por esta vez, no haya habido ni error epigráfico ni histórico.

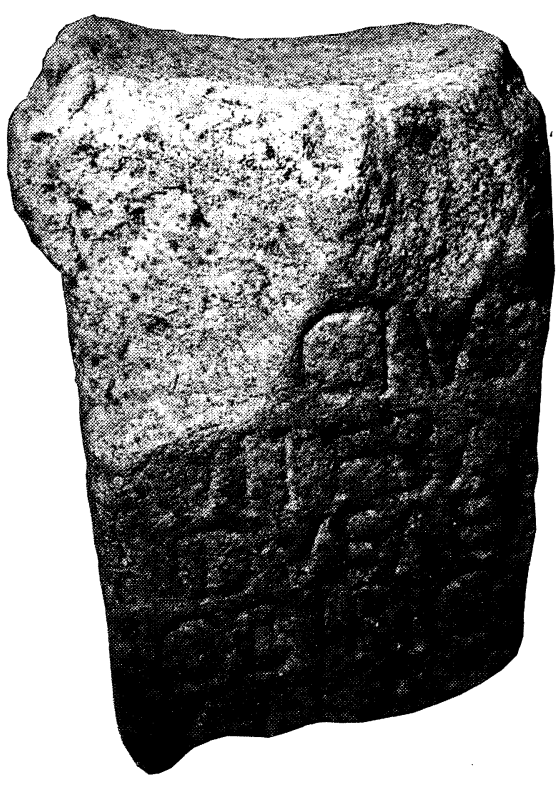

Ara recientemente aparecida.

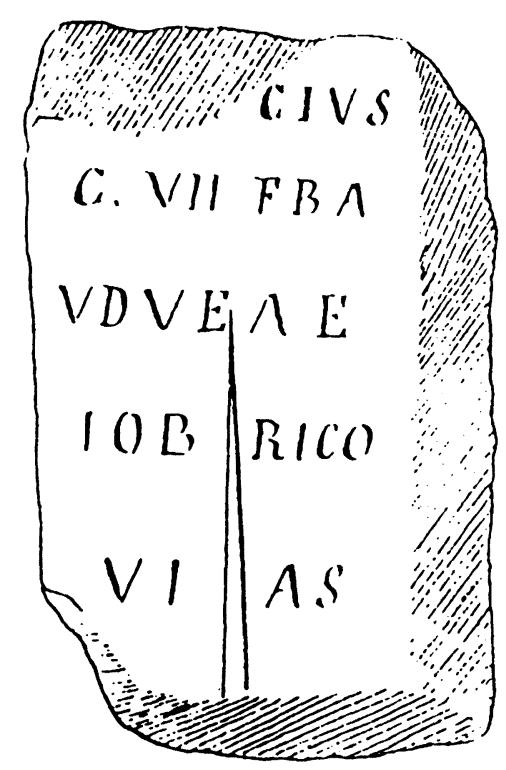

Facsímil de Barros. 